\title{
Advances in methodology and field data examples for attenuating internal multiples using the inverse scattering series
}

\author{
Jing Wu, Zhiming James Wu, Frederico Xavier de Melo* and Clément Kostov, Schlumberger
}

Copyright 2019, SBGf - Sociedade Brasileira de Geofísica

This paper was prepared for presentation during the $16^{\text {th }}$ International Congress of the Brazilian Geophysical Society held in Rio de Janeiro, Brazil, 19-22 August 2019.

Contents of this paper were reviewed by the Technical Committee of the $16^{\text {th }}$ International Congress of the Brazilian Geophysical Society and do not necessarily represent epresent any position of the sBaf, its oficers or math whent of the Brazilian Geophysical Society is prohibited.

\section{Summary}

The leading-order term in the inverse scattering series can predict internal multiples without assumptions about the subsurface. However, there are challenges related to data conditioning and computational costs in the inverse scattering series method for $2 \mathrm{D}$ or $3 \mathrm{D}$ media. We introduce an efficient data regularization strategy with the application of nearest-neighbor search and differential moveout to accommodate various acquisition situations.

We apply the angle constraints over the dip angle and opening angle to reduce the computational cost without compromising the model's quality. We propose a new method to define the optimized dip and opening angles before performing the prediction. We also propose segmented prediction by limiting the range of the multiple's generator, which can benefit the subsequent adaptive subtraction. Finally, we illustrate the proposed workflow with examples from a 2D field data set acquired in a shallow marine environment; the results demonstrate the effectiveness of the new workflow.

\section{Introduction}

Internal multiple removal remains a major challenge for both onshore and offshore plays. Traditional prediction approaches such as boundary-related assumption methods (Jakubowicz, 1998) require subsurface information about multiples generators and have limitations related to the subsurface geology.

Methods based on the inverse scattering series (ISS) have the potential to overcome limitations of such methods with respect to the subsurface structure complexity (Weglein et al., 1997). However, due to stricter input data requirements and higher computational costs, case study examples with the inverse scattering series are few in 2D and 3D (Wang and Hung, 2014), with none of the recent case studies addressing computational cost aspects.
Here, we discuss the following data conditioning and algorithm enhancement approaches that contribute to improve the inverse scattering method applications for $2 \mathrm{D}$ and 3D surveys:

- Data conditioning to meet the requirement for dense and regularly sampled data sets in sources and receivers, with sources and receivers occupying the same locations within one block;

- Limiting the integrals over frequencies according to constraints on dip and opening angles;

- Implementing segmented prediction by confining the region of multiple generators.

Finally, we illustrate the proposed method with 2D recorded data examples.

\section{Methodology}

For a 2D earth, the ISS internal multiple attenuation algorithm (Weglein et al., 1997; Kaplan et al., 2004) can be expressed as follows:

$$
\begin{aligned}
& b_{3}\left(k_{g}, k_{s}, \omega\right)= \\
& \iint_{z_{3}} d k_{1} d k_{2} \int_{-\infty}^{\infty} d z_{2} b_{1}\left(k_{1}, k_{2}, z_{2}\right) e^{-i\left(q_{1}+q_{2}\right) z_{2}} \\
& \int_{z_{2}+\varepsilon}^{\infty} d z_{1} b_{1}\left(k_{g}, k_{1}, z_{1}\right) e^{i\left(q_{g}+q_{1}\right) z_{1}} \\
& \int_{z_{2}+\varepsilon}^{\infty} d z_{3} b_{1}\left(k_{2}, k_{s}, z_{3}\right) e^{i\left(q_{2}+q_{s}\right) z_{3}}
\end{aligned}
$$

where $b_{1}$ is the input data after a constant velocity $\left(c_{0}\right)$ migration; $b_{3}$ is the predicted internal multiple; $k_{g}$ and $k_{s}$ are the horizontal wavenumbers at receiver and source sides, respectively. $\omega$ is temporal frequency. $k_{j}^{2}+q_{j}^{2}=\frac{\omega^{2}}{c_{0}^{2}}$, $j=g, s, 1,2 ; q_{i}$ is vertical wavenumber. $\varepsilon$ ensures that subevents meet a deeper-shallower-deeper relationship. The 3D equation has a similar form.

The order of depth integration in equation 1 starts with the integral over $z_{2}$ associated with downward scattering (see diagram next to equation 1). This way of computing the term $b_{3}$ was introduced by Kaplan et al. (2004) for computational efficiency reasons. In addition, equation 1 provides the option of constraining the integral limits of $z_{2}$ to specified intervals, leading to cascaded predictions as discussed for the case of 1D medium by Xavier de Melo et 
al. (2014). Here, we extend the cascaded predictions to 2D and 3D media, pursuing similar benefits as in the 1D case, and particularly for improved adaptive subtraction by applying different matching filters to models of multiples associated with different downward reflecting depth regions.

The data input to the prediction in equation 1 should be ideally free of noise, ghosts on source and receiver sides, surface multiples, and source signatures. In addition, note that equation 1 requires that data be sampled densely and regularly in source and receiver coordinates (e.g., two space coordinates for 2D media or four space coordinates for 3D media with acquisition on a horizontal surface. We fulfill this requirement by similar methods to those used for free-surface multiples predictions, including, in particular, an efficient interpolation based on weighted nearestneighbor search and differential normal moveout (Dragoset et al., 2010).

Additionally, we consider the implementation of the constraints related to dip and opening angles to reduce the computational cost (Figure 1), as proposed by Terenghi and Weglein (2012) and Ayadi and Weglein (2013).

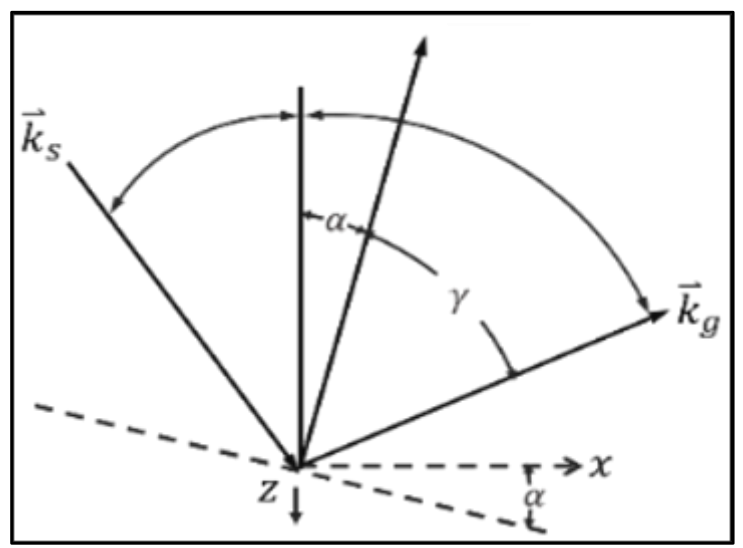

Figure 1 - Sketch of plane wave incident and reflected on a reflector with dip angle $\alpha$ and half opening angle $y$. Figure after Stolt and Weglein (2012)

\section{Numerical examples}

We discuss a numerical example with a 2D towedstreamer data set acquired in the Chukchi Sea. The survey is in shallow waters with average water-bottom depth close to $60 \mathrm{~m}$. The nominal source array depth is $6.1 \mathrm{~m}$ and the nominal streamer depth is $12 \mathrm{~m}$. A stack of the $2 \mathrm{D}$ line after attenuating noise, ghosts, and surface multiples is shown in Figure 2, illustrating the geology complexity with a potentially large number of significant internal multiples generators. Highlighted boxes define the area of study when determining angle constraints parameters (in blue) and when presenting examples for segmented prediction and sequential subtraction (in red).

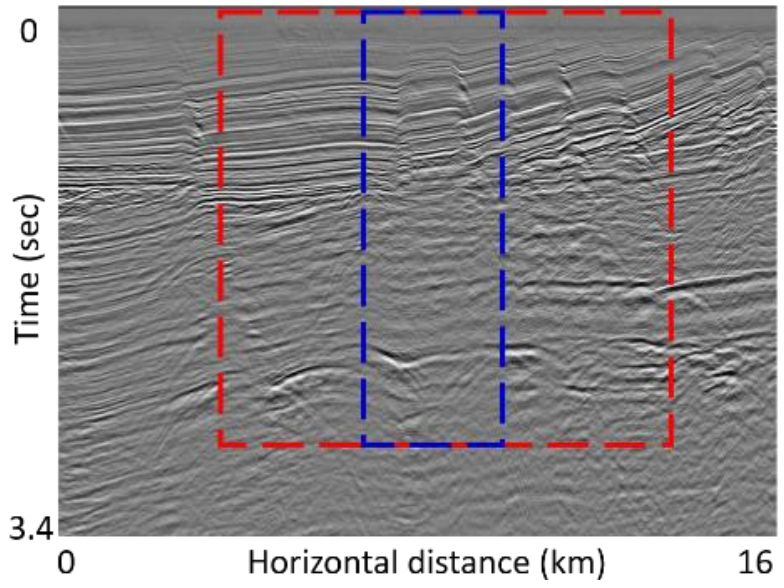

Figure 2 - Stack profile of Chukchi data. Blue box defines the area of study related to angle constraint. Red box shows the area used to highlight the segmented prediction and sequential prediction approach.

\section{Numerical examples: data regularization}

The streamer geometry only allows us to locate receivers at one side of the source and does not sample the verynear offset. However, the ISS requires collocated source and receivers for every processing block (as shown in Figure 3), within which the receiver stations are stationary while sources move from one receiver location to the next. We fulfill this condition by implementing an on-the-fly interpolation. The sampling parameters before and after data conditioning are listed in Table 1.

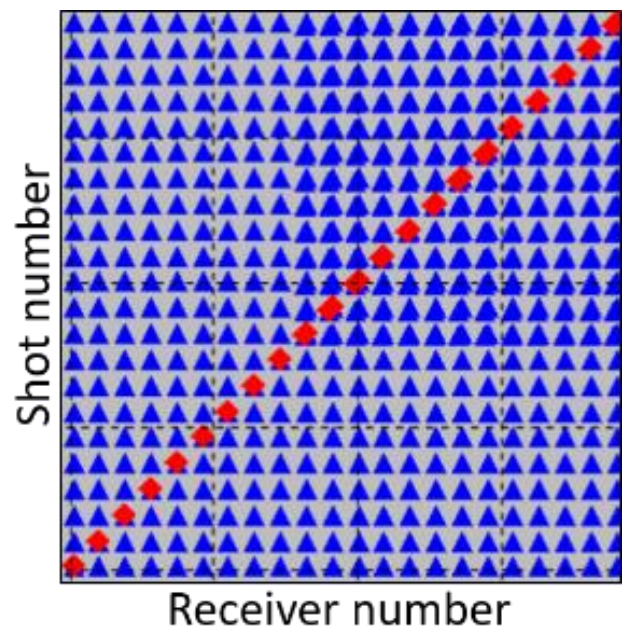

Figure 3 - Sketch of desired 2D geometry for ISS prediction shown by shot (red) and receiver (blue) numbers.

\section{Numerical examples: angle constraints}

$\mathrm{Next}_{1}$ we analyze the ISS predictions and computational cost by keeping the range of half opening angle with $\left[0^{\circ}\right.$, $80^{\circ}$ ], while changing only the range of dip angle. We do not aggressively limit the opening angle, as a wide range of 
opening angles is required to fully express the reflected and diffracted events in shallow-water data. Figures 4 a to
Arrows point to visible places where angle constraints negatively affect the predicted model.

\begin{tabular}{|l|l|l|}
\hline Parameters & Before interpolation & After interpolation \\
\hline Shot interval & $26.67 \mathrm{~m}$ & $18 \mathrm{~m}$ \\
\hline Receiver interval & $26.67 \mathrm{~m}$ & $18 \mathrm{~m}$ \\
\hline Gather range & {$[186.66 \mathrm{~m}, 3200 \mathrm{~m}]$} & Block size $6400 \_\mathrm{m}$ with fixed receivers and moving sources ${ }_{i}^{;} ;$offset range varies \\
\hline
\end{tabular}

Table 1 - Key parameters for the test data set before and after geometry regularization.

$4 \mathrm{~d}$ are prediction models (stack profiles) with dip angles up to $90^{\circ}, 50^{\circ}, 30^{\circ}$, and $10^{\circ}$, respectively. Figure 4 a shows the best result from this method. The differences between Figures $4 \mathrm{~b}$ and $4 \mathrm{a}$, as well as between $4 \mathrm{c}$ and $4 \mathrm{a}$, are very small.
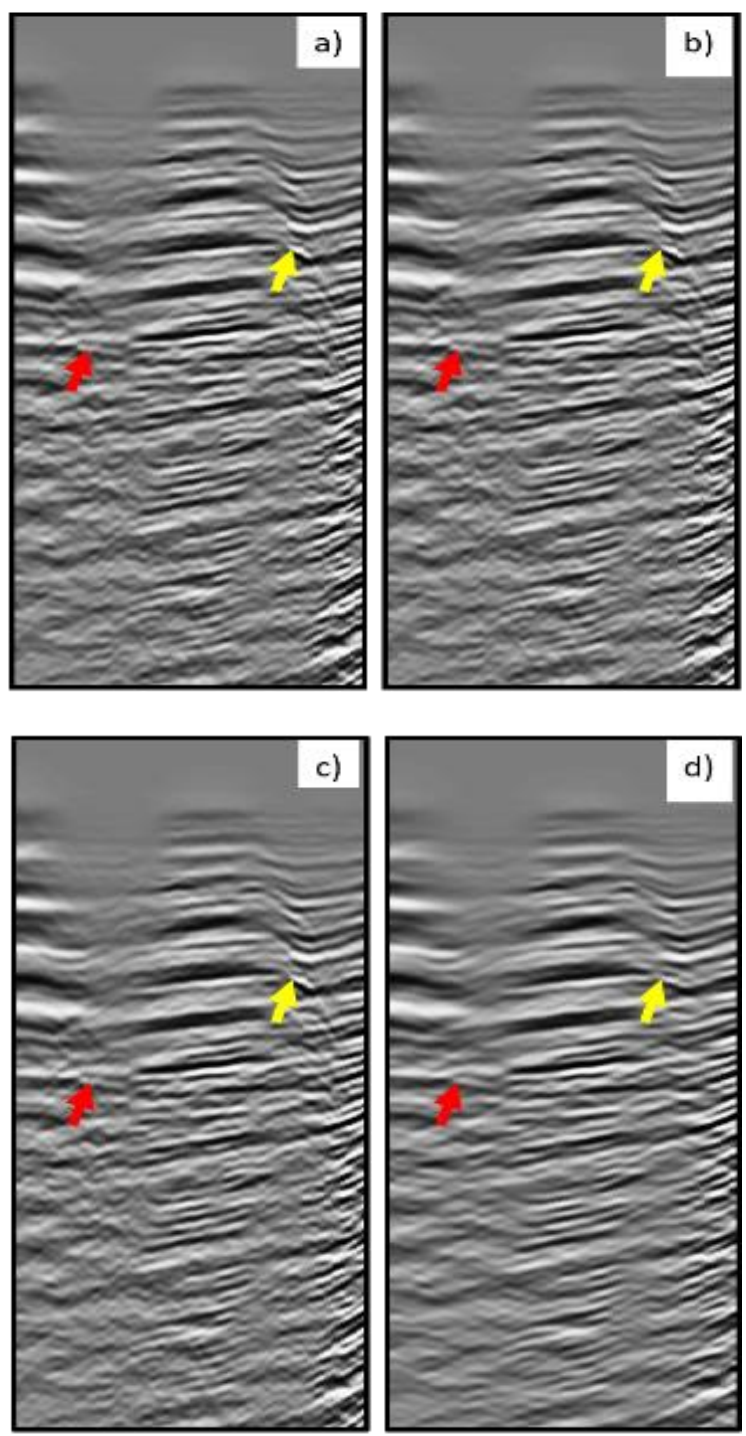

Figure 4-Stacks of predictions with dip angle constraints. Maximum dip up to a) $90^{\circ}$, b) $50^{\circ}$, c) $30^{\circ}$, and d) $10^{\circ}$.
Figure 5 shows a quantitative comparison between the stacked predicted models from Figure 4 by displaying the root-mean-squared (RMS) amplitudes of the differences between the constrained (Figures $4 \mathrm{~b}$ to $4 \mathrm{~d}$ ) and unconstrained (Figure 4a) models $\operatorname{RMS}\left(A_{i}-A_{90}\right) /$ $R M S\left(A_{90}\right)$. RMS results are normalized by the unconstrained models and are represented by the blue curve in Figure 5, indicating that selecting the maximum dip angle wider than $20^{\circ}$ leads to an error smaller than $5 \%$. The orange curve is the runtime ratio (run time with different maximum angles/runtime with full dip angle), which shows that the algorithm can be speeded up by 1.5 $\sim 2.5$ times by reducing the maximum dip angle to be $20^{\circ}$ $\sim 30^{\circ}$. These two curves show that properly incorporating angle constraints to the algorithm can improve the efficiency without sacrificing the model's quality.

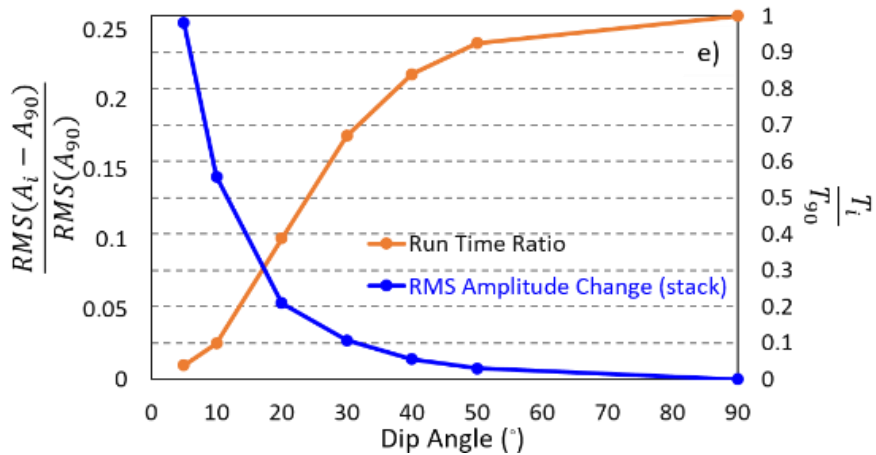

Figure 5-Quality, cost $t_{2}$ and effectiveness analysis: $A_{i}$ are the RMS amplitudes; $T_{i}$ is run time. Curves help define an optimal constraint value that maximizes efficiency.

We propose a simple and straightforward method to optimally select dip and opening angle parameters by looking at the recorded data with different angle constraints before prediction. As an example, we kept the half-opening angle up to $80^{\circ}$, while varying the upper limit of the dip angle, as shown on stacked images (Figures $6 a-6 d$ ).

Applying the normalized RMS difference on each dip angle outcome (Figure 7), we can observe the same trend of the curve in Figure 5 and define an optimal angle trade-off between quality and efficiency. 

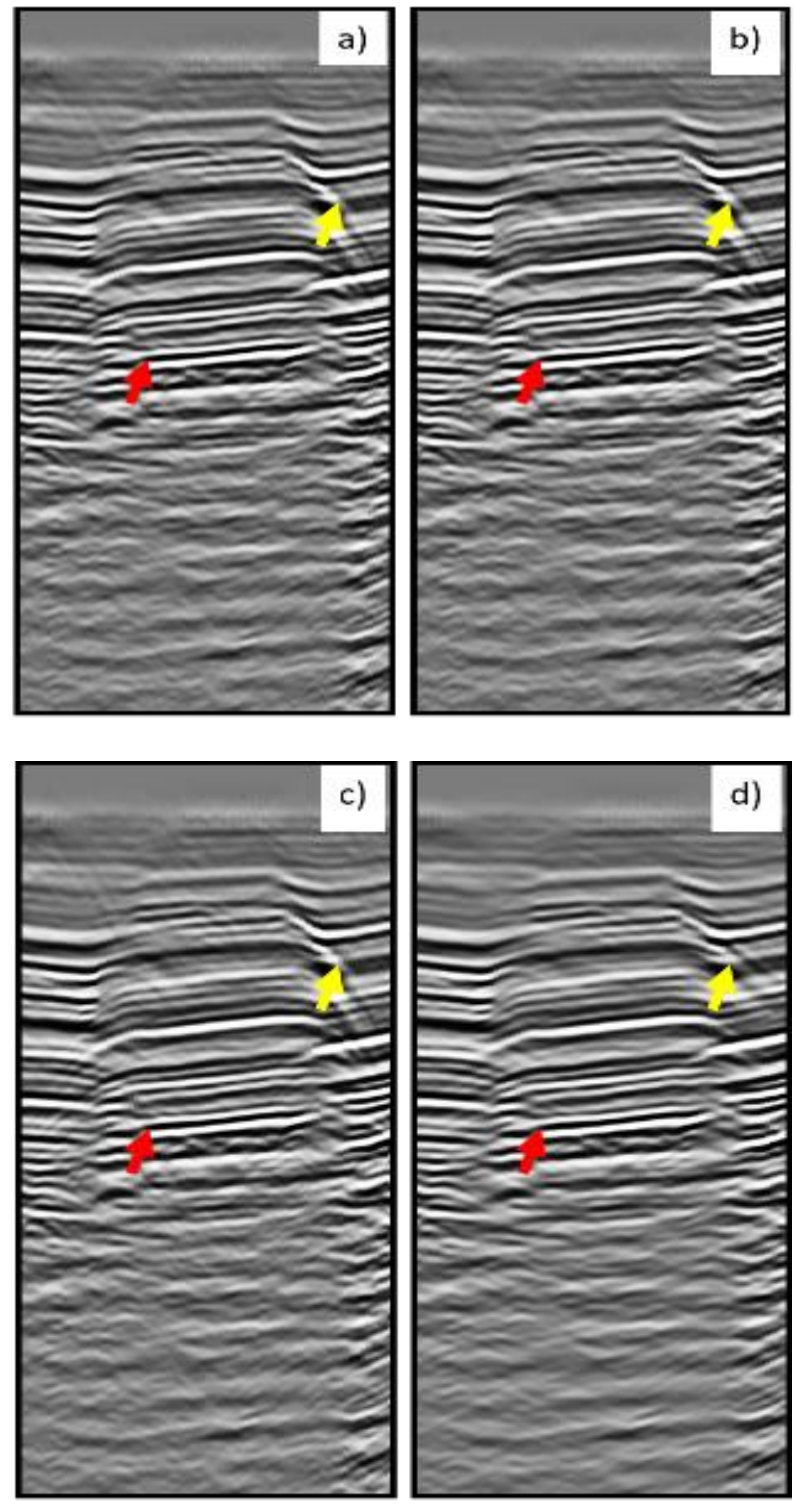

Figure 6 - Stack after decomposing input data with dip angle limits. Max dip up to a) $90^{\circ}$, b) $50^{\circ}$, c) $30^{\circ}$, and d) $10^{\circ}$. Arrows point to visible places where angle constraints negatively affect the predicted model.

\section{Numerical examples: segmented prediction and sequential adaptive subtraction}

Next, a new workflow using predicted models for three specified depth intervals is compared to a reference workflow using only one model predicted from generators in a single depth interval chosen as the union of the three previously specified intervals.

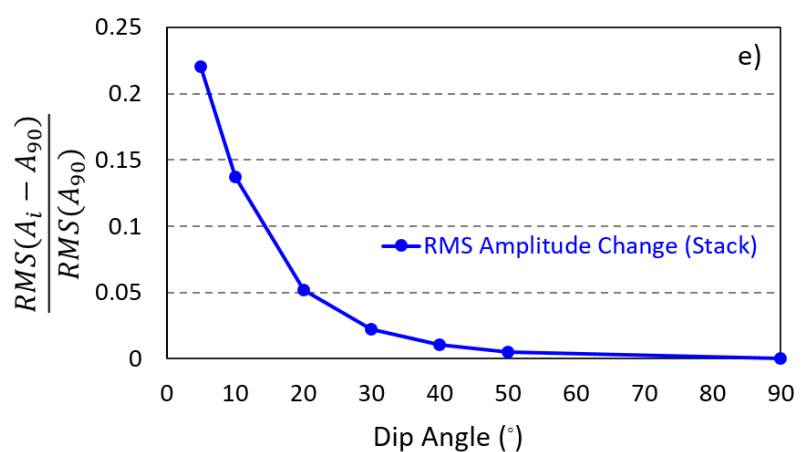

Figure 7 - Quality analysis based on angle constraint simulation using the recorded data only: $A_{i}$ values are the RMS amplitudes.

We define three-generator regions in pseudo-depth (after water-speed migration), with $300 \mathrm{~m}$ for each from $0 \mathrm{~m}$ down to $900 \mathrm{~m}$. We predict a subset of internal multiples for each window, followed with sequential adaptive subtractions of the predicted models; i.e., we conduct the subtraction with input data and the model from the first window, then the result is used as input for subtracting the model from the second widow, until down to the deepest one.

Figure $8 \mathrm{a}$ is input data. The final subtraction result is shown in Figure 8b. For the reference result, we choose a large window down to $900 \mathrm{~m}$ and we perform a single subsequent adaptive subtraction. The corresponding subtraction result is shown in Figure 8c. As highlighted in Figures $8 \mathrm{a}$ to $8 \mathrm{c}$ with arrows and boxes, the internal multiple is effectively removed with the new strategy; whereas, the residual multiple remains significant in the reference result. These results illustrate the benefits of splitting the predicted multiples by models corresponding to specified smaller depth intervals.

\section{Conclusions}

We presented an effective inverse scattering series workflow for predicting internal multiples for cases of $2 \mathrm{D}$ or 3D scenarios.

The workflow addresses the data sampling limitations, data conditioning requirements, as well as the high computational costs. Specifically, we make use of neighboring trace searches and differential NMO corrections in preparing the input data; we impose constraints on the dip angles, and compute ISS models as functions of specified depth intervals containing downward reflections. 


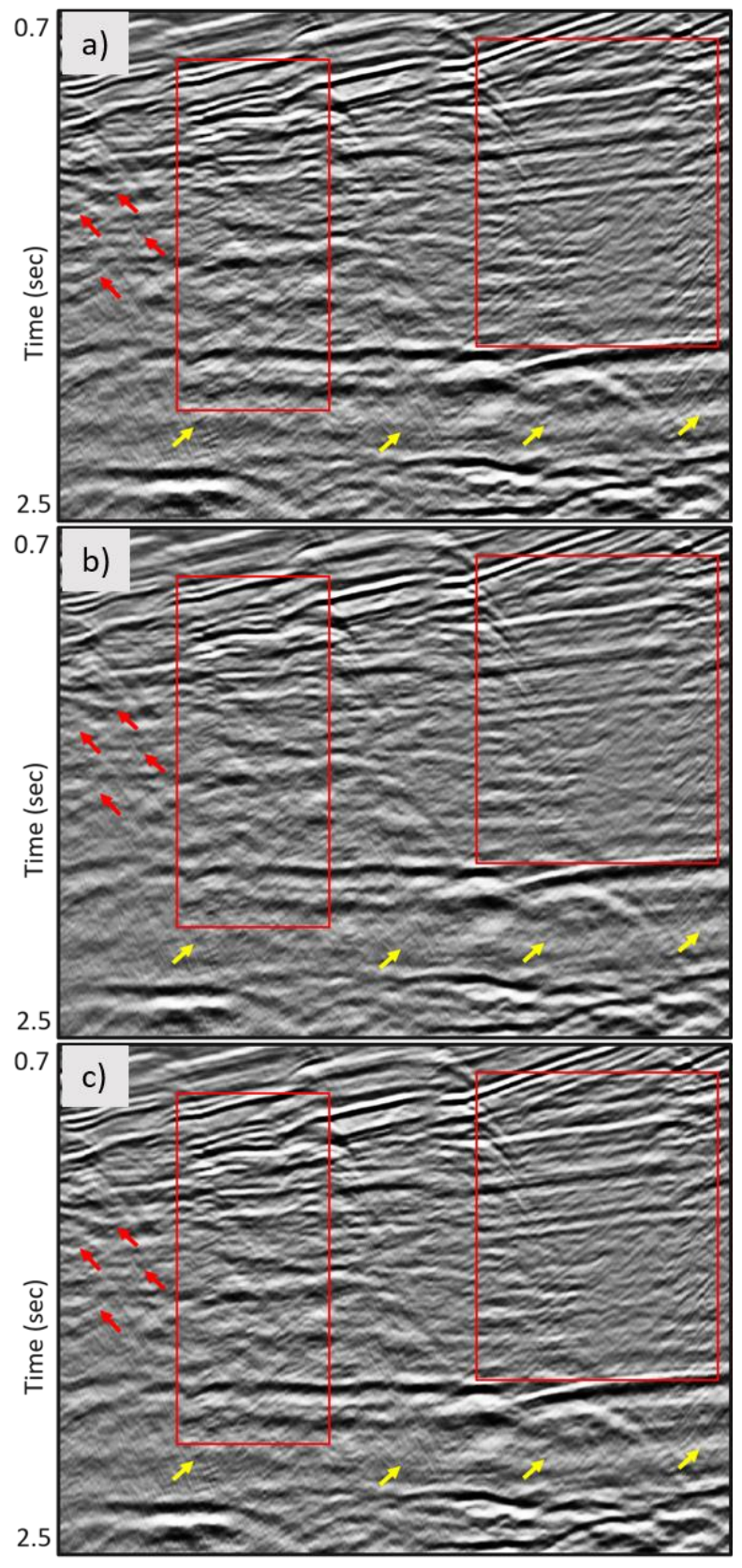

Figure 8 - ISS internal multiple prediction and attenuation results. a) input data stack; b) subtraction result with new flow; c) subtraction result with the legacy flow.

The proposed workflow is illustrated with examples from a 2D data set acquired in shallow water. In this example, many primaries act as significant multiples generators and, hence, the ISS method is preferred to the boundary-related prediction method because the ISS method requires no selection of multiples generators.

We show improved subtraction results by the new approach versus a conventional ISS prediction workflow where all internal multiples are part of the same model without distinction as a function of the downward generator. In addition, we report computational savings due to combining angle constraints and propose a new method to select the optimized dip angle and opening angle.

\section{Acknowledgments}

We thank WesternGeco Multiclient for permission to use their data and Schlumberger management for permission to present.

\section{References}

AYADI, $H$. and WEGLEIN, A.B. Time saving method based on angular quantities applied to an internal multiple attenuation algorithm: Fundamental concept, development, and numerical analysis. 83rd Annual International Meeting, SEG, Expanded Abstracts, 41624166, 2013

DRAGOSET, B., VERSCHUUR, D.J., MOORE, I., and BISLEY, R. A perspective on 3D surface-related multiple elimination. Geophysics, 75, 75A245-75A261, 2010.

JAKUBOWICZ, H. Wave equation prediction and removal of interbed multiples. 68th Annual International Meeting, SEG, Expanded Abstracts, 1527-1530, 1998.

KAPLAN, S.T., INNANEN, K.A., OTNES, E. and WEGLEIN, A.B. Internal multiple attenuation codedevelopment and implementation. M-OSRP Annual Report, 83-102, 2004.

STOLT, R. H., and WEGLEIN, A.B. Seismic imaging and inversion: Volume 1. Application of Linear Inverse Theory. Cambridge University Press, 2012.

TERENGHI, P., and WEGLEIN, A. B. ISS internal multiple attenuation with angle constraints. M-OSRP Annual Report, 242-266, 2012.

WEGLEIN, A.B., GASPAROTTO, F.A., CARVALHO, P.M., and STOLT, R.H. An inverse-scattering series method for attenuating multiples in seismic reflection data. Geophysics, 62, 1975-1989, 1997.

WANG, M., and HUNG, B. 3D Inverse Scattering Series Method for Internal Multiple Attenuation. 76th Conference and Exhibition, EAGE, Extended Abstracts, We E102 06, 2014.

XAVIER DE MELO, F., IDRIS, M., WU, Z.J., and KOSTOV, C. Cascaded internal multiple attenuation with inverse scattering series: Western Canada case study. 84th Annual International Meeting, SEG, Expanded Abstracts, 4113-4117, 2014. 